\title{
The Glutamic Acid-Rich Protein Is a Gating Inhibitor of Cyclic Nucleotide-Gated Channels
}

\author{
Stylianos Michalakis, ${ }^{1,2 *}$ Xiangang Zong, ${ }^{1,2 *}$ Elvir Becirovic, ${ }^{1,2 *}$ Verena Hammelmann, ${ }^{1,2}$ Thomas Wein, ${ }^{2}$ \\ Klaus T. Wanner, ${ }^{2}$ and Martin Biel ${ }^{1,2}$ \\ ${ }^{1}$ Munich Center for Integrated Protein Science and ${ }^{2}$ Department of Pharmacy-Center for Drug Research, Ludwig-Maximilians-Universität München, \\ D-81377 München, Germany
}

The cyclic nucleotide-gated (CNG) cation channel of rod photoreceptors is a heterotetramer consisting of homologous CNGA1 and CNGB1 a subunits. While CNGA1 is indispensable for channel activation, the specific role of CNGB1a in this process has remained elusive. Here, we show that the N-terminal glutamic acid-rich protein (GARP) domain of CNGB1a and soluble GARP2, which corresponds to the proximal portion of the GARP domain, act as autoinhibitory domains that decrease the opening probability of the CNG channel. In the presence of mutations that structurally impair the cyclic nucleotide-binding domain (CNBD) of CNGB1a, the GARP domain completely abolishes channel activity. In agreement with an inhibitory function of GARP, the activity of mutant CNG channels could be fully restored by deletion of the GARP domain. We identified two sequences within the GARP domain that confer most of the inhibitory effect and demonstrate that the profound inhibition imposed by the GARP domain is caused by direct and autonomous protein-protein interaction with the CNG channel complex. In wild-type rod CNG channels, this inhibitory effect can be relieved by binding of cGMP to the CNBD of CNGB1a. In conclusion, we propose that the $\mathrm{N}$ terminus of CNGB1a and soluble GARPs act as molecular gate keepers that control the activation of heteromeric rod CNG channels. Our results suggest that the GARP domain has evolved in rod photoreceptors to reduce current noise resulting from openings of CNG channels in the absence of cGMP.

\section{Introduction}

The rod cyclic nucleotide-gated (CNG) channel is a heterotetramer composed of three CNGA1 and one CNGBla subunit (Weitz et al., 2002; Zheng et al., 2002; Zhong et al., 2002). Both types of subunits contain six transmembrane helices, an ionconducting hairpin, and a cyclic nucleotide-binding domain (CNBD) in the C terminus. Unlike CNGA1, CNGB1a does not form functional channels when expressed alone in heterologous systems. However, when assembled with CNGA1, it confers several properties that are characteristic of the native rod channel, including single-channel flickering, inhibition by $\mathrm{Ca}^{2+} /$ calmodulin, and micromolar sensitivity to L-cis-diltiazem (Chen et al., 1993; Körschen et al., 1995). In addition, CNGB1a affects $\mathrm{Ca}^{2+}$ permeation and blocking properties (Chen et al., 1993; Körschen et al., 1995) and is crucial for CNG channel trafficking to rod outer segments (Hüttl et al., 2005; Zhang et al., 2009). CNGB1a has a unique bipartite structure consisting of the "channel domain" comprising the C-terminal half of the subunit and a large

Received Sept. 9, 2010; revised 0ct. 14, 2010; accepted 0ct. 22, 2010.

This work was supported by the Deutsche Forschungsgemeinschaft (Mi 1238/1-2). We thank Heidi Sebald, Jennifer Schmidt, Stephanie Stark, and Berit Noack for excellent technical support, Dr. Steven Siegelbaum (Columbia University) for helpful discussion, Dr. Robert S. Molday (University of British Columbia) for the gift of PPc6N antibody, and Dr. U. Benjamin Kaupp (Caesar Bonn) for the gift of bCNGA1.

*S.M., X.Z., and E.B. contributed equally to this work.

Correspondence should be addressed to Martin Biel, Department Pharmazie-Pharmakologie für Naturwissenschaften, Ludwig-Maximilians-Universität München, Butenandtstr. 5-13, D-81377 München, Germany. E-mail: mbiel@cup.uni-muenchen.de.

DOI:10.1523/JNEUROSCI.4735-10.2011

Copyright $\odot 2011$ the authors $\quad$ 0270-6474/11/310133-09\$15.00/0
$\mathrm{N}$-terminal extension called glutamic acid-rich protein (GARP) domain (Körschen et al., 1995). Remarkably, the complete and the N-terminal half of the GARP domain are also expressed in rod photoreceptors as soluble proteins, designated GARP1 and GARP2, respectively (Biel et al., 1993; Körschen et al., 1995; Colville and Molday, 1996). Although shorter isoforms of CNGB1 are expressed in other neurons (e.g., CNGB1b in olfactory sensory neurons), all of these isoforms lack the $\mathrm{N}$-terminal GARP domain. Thus, the GARP domain seems to be specific for rod photoreceptors.

Several functions have been proposed for soluble and channel-attached GARP domains, including tethering CNG channels to the disc rim of rod outer segments (Batra-Safferling et al., 2006), regulation of PDE6 activity (Körschen et al., 1999; Pentia et al., 2006), or serving as high-capacity $\mathrm{Ca}^{2+}$ buffer (Haber-Pohlmeier et al., 2007). It is unknown, however, whether GARP domains are involved in principal CNG channel gating and to what extent the CNBD of the CNGB1a subunit contributes to the activation process of the heteromeric rod CNG channel. In this study, we set out to address these important questions. To this end, we functionally analyzed a point mutation in the human CNGB1 gene that leads to a glycine-valine exchange at position 993 in the CNBD of CNGB1a (later on referred to as "GV" mutation). The GV mutation was identified in a French family suffering from retinitis pigmentosa (RP) (Bareil et al., 2001), a progressive degenerative eye disease that is characterized by loss of rod function. In agreement with the severe clinical phenotype, we found that heteromeric CNGA1/CNGB1aGV channels are functionally inactive. Surprisingly, however, the dominant- 
negative effect of the GV mutation was fully removed when the GARP domain was deleted. We show that the inhibitory action of GARP is transferable to different CNG channel types and that it probably results from direct interaction between the GARP domain and the CNG channel complex. Our study indicates that the presence of a functional CNBD in the CNGB1 subunit is indispensable to relief tonic inhibition imposed by the GARP domain.

\section{Materials and Methods}

Molecular biology. The bovine CNGA1 (bCNGA1) clone (Kaupp et al., 1989) was a kind gift from Dr. U. B. Kaupp (Caesar, Bonn, Germany). Rat CNGB1a and CNGB1b and GARP2 were previously described (Sautter et al., 1997, 1998). Peripherin-2 (Prph2) was PCR amplified from mouse retinal cDNA (Thermoscript Kit, Invitrogen) and subcloned into pcDNA3.1/myc-His C (Invitrogen). For Förster resonance energy transfer (FRET) experiments, eCFP was fused to the $\mathrm{C}$ terminus of bovine CNGA1 and eYFP was fused to the $\mathrm{N}$ terminus of wild-type or mutant rat CNGB1a. All cDNAs were subcloned into pcDNA3, pcDNA3.1/myc-His, or pIRES2-EGFP vectors for expression in HEK293 cells. Site-directed mutagenesis was performed by standard procedures. All constructs were sequenced before functional analysis.

Biochemical assays. Membrane or whole-cell proteins were isolated from transfected HEK293 cells as described previously (Much et al., 2003). Coimmunoprecipitation was performed using protein G Dynabeads (Invitrogen). Briefly, $5 \mu \mathrm{g}$ of antibody [anti-myc clone 9B11 (New England Biolabs) or anti-CNGB1 (Hüttl et al., 2005)] and PBS were added to the Dynabeads to a final volume of $500 \mu \mathrm{l}$. This solution was rotated for $30 \mathrm{~min}$ at $4^{\circ} \mathrm{C}$. Subsequently, the beads were separated magnetically followed by a wash step with PBS. One milligram of protein lysate was added to the beads, and the reaction was filled up with PBS to a final volume of $500 \mu \mathrm{l}$. This suspension was rotated for $30 \mathrm{~min}$ at $4^{\circ} \mathrm{C}$ followed by three washing steps with PBS. After the last wash, beads were resuspended in $6 \times$ Lämmli buffer (without reducing reagents) and were incubated at $70^{\circ} \mathrm{C}$ for $15 \mathrm{~min}$. The proteins (supernatant) were separated on the SDS-PAGE gel and then processed for Western blotting. For in vivo experiments $1.5 \mathrm{mg}$ of Triton $\mathrm{X}-100$ lysates from $\mathrm{CNGB}^{+/+}$and $\mathrm{CNGB1}^{-/-}$mice (Hüttl et al., 2005) were incubated with $10 \mu \mathrm{g}$ of anti$\mathrm{CNGB} 1$ antibody for $2 \mathrm{~h}$ at $4^{\circ} \mathrm{C}$. Subsequently, the cleavable crosslinking reagent DTSSP [3,3'-dithiobis(sulfosuccin-imidylpropionate), $5 \mathrm{~mm}$, Thermo Scientific] was added to the mixture and the reaction was incubated for additional $2 \mathrm{~h}$ at $4^{\circ} \mathrm{C}$. The reaction was stopped by $50 \mathrm{~mm}$ Tris, $\mathrm{pH}$ 7.5. Fifty microliters of protein G Dynabeads (Invitrogen) were added and the solution was incubated overnight at $4^{\circ} \mathrm{C}$. After washing the beads, before the loading on the SDS-PAGE gel, DTSSP crosslinks were cleaved by boiling $\left(5 \mathrm{~min}, 100^{\circ} \mathrm{C}\right)$ the samples in SDS-PAGE loading buffer containing $\beta$-mercaptoethanol and DTT.

Cell surface biotinylation was performed using $0.8 \mathrm{~mm}$ non-cellpermeable sulfo-NHS-SS-biotin (Pierce) in PBS for $30 \mathrm{~min}$ at $4^{\circ} \mathrm{C}$ as described by Luna (2001). The reaction was stopped with $10 \mathrm{~mm}$ glycine. In control experiments, the labeling procedure was repeated for $1 \mathrm{~h}$ at $37^{\circ} \mathrm{C}$, which results in biotinylation of intracellular proteins in addition to cell surface proteins. After solubilization [Triton X-100 (Much et al., 2003)], biotinylated proteins were precipitated on NeutrAvidin agarose resin (Pierce), treated with DTT to remove the biotin label, separated on a 7\% SDS-PAGE, and then processed for Western blotting. We used the following antibodies for Western blotting: anti-ATPase (1:1000, clone $\alpha 6 \mathrm{~F}$, developed by D.M. Fambrough, obtained from the Developmental Studies Hybridoma Bank), anti-CNGB1 (1:10,000) (Hüttl et al., 2005), anti-GFP (1:1000, Clontech), anti-myc (1:1000, clone 9B11, New England Biolabs), PPc6N (1:1000) (Colville and Molday, 1996), and antiTubulin (1:400, Dianova).

FRET. HEK293 cells were transfected with CNGA1-CFP or soluble CFP (negative control) and wild-type or mutant YFP-CNGB1a. A CFPYFP tandem construct served as FRET-positive control (Griessmeier et al., 2009). Fluorescent images were captured $48-72 \mathrm{~h}$ after transfection on an Axioplan 2 microscope (Zeiss) equipped with a Plan Neofluar $40 \times$ objective (numeric aperture 0.75), a HMRc ccd camera, and an AttoArc HBO100 mercury lamp illumination unit. Three fluorescent images per cell were collected using the CFP HC filter, YFP HC filter, and FRET CFP-YFP HC filter sets (Semrock). Fluorescence images were processed using the FRET plus macro (Zeiss), and the FRET efficiency was calculated as N-FRET using the method described by Xia and Liu (2001).

Electrophysiology. Macroscopic currents and single-channel currents were recorded from inside-out patches excised from HEK293 cells by using an Axopatch 200B amplifier and pCLAMP10 software (Molecular Devices). The pipette and bath solution were identical and contained 140 $\mathrm{mm} \mathrm{NaCl}, 5.0 \mathrm{~mm} \mathrm{KCl}, 10 \mathrm{~mm}$ HEPES, and $1.0 \mathrm{~mm}$ EGTA (divalent cation-free solution, $\mathrm{pH} 7.4$ with $\mathrm{NaOH}$ ). For measurement of the currents in the presence of extracellular $\mathrm{Ca}^{2+}$, the following solution was used in the pipette: $140 \mathrm{~mm} \mathrm{NaCl}, 5.0 \mathrm{~mm} \mathrm{KCl}, 2.0 \mathrm{~mm} \mathrm{CaCl}_{2}, 1.0 \mathrm{~mm}$ $\mathrm{MgCl}_{2}$, and $10 \mathrm{~mm}$ HEPES, $\mathrm{pH}$ 7.4. A multibarreled perfusion pipette placed $200 \mu \mathrm{m}$ away from the patch was used to switch the superfusion solution. The membrane potential was held at $0 \mathrm{mV}$ and stepped to \pm 80 $\mathrm{mV}$ for $1.8 \mathrm{~s}$. Capacitative transients and leak currents were subtracted using currents recorded under the superfusion with solution without agonist. Data were filtered at $2 \mathrm{kHz}$, digitized at $20 \mathrm{kHz}$, and analyzed with Clampfit and Origin software. Dose-response curves for cGMP and cAMP were generated by measuring the current response at $+80 \mathrm{mV}$ and fitted with the Hill equation: $I / I_{\max }=(C)^{\nu} /\left((C)^{\nu}+K_{1 / 2}^{\nu}\right)$, where $I_{\max }$ is the current at a saturating concentration $(1 \mathrm{mM})$ of cGMP, $(C)$ is the cyclic nucleotide concentration, $K_{1 / 2}$ is the activation constant, and $n$ is the Hill coefficient. Maximal channel open probability $\left(P_{\max }\right)$ was determined from the ratio of currents at saturating cGMP $(1 \mathrm{mM})$ in the absence and the presence of $1 \mu \mathrm{M} \mathrm{Ni}^{2+} \cdot \mathrm{Ni}^{2+}$ was shown to potentiate currents induced by cGMP (Ildefonse et al., 1992; Gordon and Zagotta, 1995; Pagès et al., 2000), and the ratio described above was shown to be almost indistinguishable from the $P_{\max }$ values obtained from singlechannel measurements (Sunderman and Zagotta, 1999). $\mathrm{KCl}$ or $\mathrm{NaCl}$ with $<0.01 \mathrm{ppm} \mathrm{Ni}^{2+}$ was used and HEPES concentration was $5 \mathrm{~mm}$. EGTA/EDTA were omitted from the extracellular solution. The pipette solution contained $200 \mathrm{~mm}$ EDTA and $500 \mathrm{~mm}$ niflumic acid. All experiments were done at room temperature $\left(20-22^{\circ} \mathrm{C}\right)$.

Molecular modeling. The crystal structure of the C-terminal fragment of SpIH (pdbid = 2Q0A) (Flynn et al., 2007) was used as structural template for building the tetrameric 3D structures of wild-type and G993V mutant of human CNGB1a. Twenty structures of the wild-type and the G993V mutant were built using the default MODELLER (Eswar et al., 2007) parameter settings. The structures with the lowest value of the MODELLER objective function were further refined using GROMACS (Van Der Spoel et al., 2005). The structures were soaked in a periodic box of SPC water (Berendsen et al., 1981) and energy minimized using the steepest descent method. Negatively charged chloride ions were used to make the full system (protein and water) zero charged. For electrostatics, particle-mesh Ewald PME method was used (Darden et al., 1993). A short simulated annealing protocol with heating up the systems from $0 \mathrm{~K}$ to $310 \mathrm{~K}$ in $5 \mathrm{ps}$, equilibration for $10 \mathrm{ps}$, and cooling down from $310 \mathrm{~K}$ to $0 \mathrm{~K}$ in $5 \mathrm{ps}$, was executed to avoid overlapping atoms and misfolded regions from structure building. During all simulations, $\mathrm{C} \alpha$ atoms of residues close to the mutated glycine 993 and the cGMP binding site were allowed to move freely (residues 968-969, 972-974, 991-995, 1028-1032, 1041-1044, 10681069,1083 , and 1086). All other C $\alpha$ atoms were restrained to their position to avoid unfolding during simulation.

Statistics. All values are given as mean $\pm \mathrm{SE}$, and $n$ is the number of experiments. An unpaired Student's $t$ test was performed for the comparison between two groups. Values of $p<0.05$ were considered significant.

\section{Results \\ The GV mutation impairs cGMP-dependent channel activation}

The CNBD is a highly conserved protein domain that contains three $\alpha$-helices $(\alpha \mathrm{A}-\mathrm{C})$ and eight $\beta$-strands forming an antiparallel $\beta$-roll (total length $123 \mathrm{aa}$ ). The GV mutation in the CNBD of CNGB1a refers to a glycine residue in the $\beta 2-\beta 3$ connecting loop that is strictly conserved throughout evolution (Fig. $1 A)$. The available crystal structures of CNBDs indicate that the 


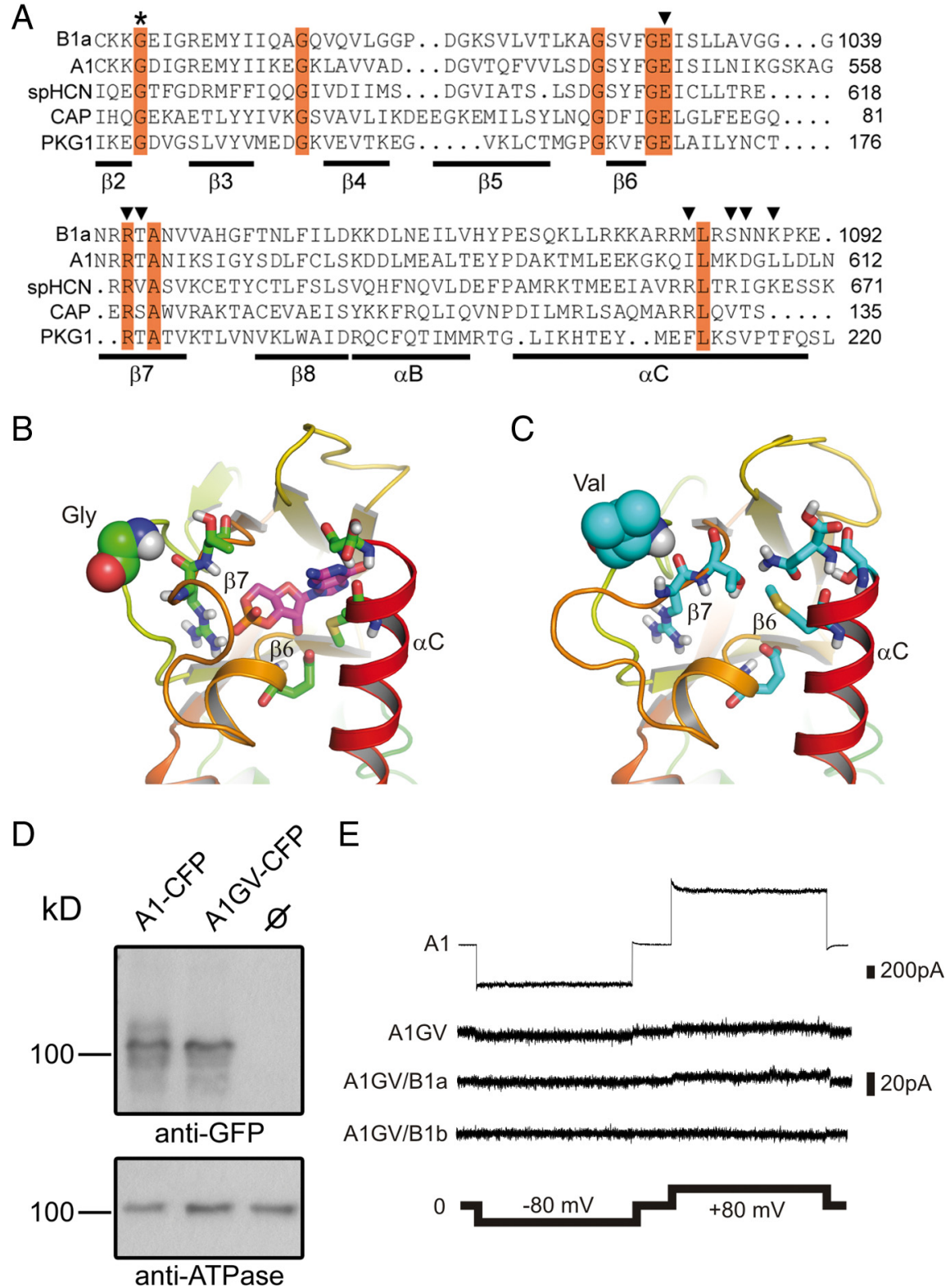

Figure 1. Effects of the GV mutation on the structure of the CNBD and CGMP-dependent channel activation. $\boldsymbol{A}$, Sequence alignment of the $\beta 2-\alpha$ C region of the CNBD of human CNGB1a, human CNGA1, sea urchin HCN1, Escherichia coli CAP, and human PKG1. Invariant residues are highlighted in red. The invariant glycine residue within the $\beta 2-\beta 3$ loop that is mutated to valine (GV mutation) in the (NGB1a subunit of RP patients is marked with an asterisk. Residues identified in the crystal structure of HCN channel CNBDs to participate in cyclic nucleotide-binding are marked with arrowheads (Zhou and Siegelbaum, 2007). B, C, Predicted structure of the CGMP binding pocket of CNGB1a $(\boldsymbol{B})$ and CNGB1aGV $(\boldsymbol{C})$. The sequences were threaded onto the crystal structure of the CNBD of spHCN (Flynn et al., 2007) by using MODELLER (Eswar et al., 2007) and analyzed using molecular dynamics simulations (see Materials and Methods). Only one monomer is shown for clarity. The glycine and the valine residue in the $\beta 2-\beta 3$ loop of CNGB1a and CNGB1aGV, respectively, are represented as space-filling spheres. Residues in the $\beta 6$ and $\beta 7$ strands as well as in the $\alpha$ Chelix participating in CGMP binding are shown as sticks. In $\boldsymbol{B}$, a cGMP molecule (shown as magenta sticks) was pasted onto the modeled structure based on information from the CGMP-bound spHCN CNBD crystal structure (Flynn et al., 2007). D, CNGA1GV is expressed at the plasma membrane of transfected HEK293 cells. Top, Western blot of membranes from HEK293 cells transfected with CNGA1-CFP or CNGA1GV-CFP, respectively. The blot was probed with anti-GFP antibody. Bottom, Membranes probed against the ATPase $\alpha$ subunit. CNBD, cyclic nucleotide-binding domain. $\boldsymbol{E}$, Representative current traces elicited by $1 \mathrm{~mm}$ cGMP and voltage steps (2 s each) to $-80 \mathrm{mV}$ and $+80 \mathrm{mV}$ in excised patches from HEK293 cells expressing CNGA1 (A1) or CNGA1GV (A1GV), CNGA1GV/CNGB1a (A1GV/B1a), and CNGA1GV/CNGB1b (A1GV/B1b).

glycine is not directly involved in cGMP binding but that it is essential for the integrity of the overall fold of the CNBD (Shabb and Corbin, 1992). We performed a molecular dynamics (MD) simulation to model conformational changes within the CNBD of CNGB1a that may result from the GV mutation (Fig. $1 B, C$ ).
As a template, we used coordinates from the crystal structure of the CNBD of the sea urchin HCN channel (Flynn et al., 2007). We found that the GV mutation induces major structural alterations in the CNBD (Fig. 1C) by forcing the backbone dihedral angles of the mutated valine to preferred regions in the Ramachandran plot $\left(\varphi=70^{\circ}, \psi=80^{\circ}\right.$ for valine versus $\varphi=130^{\circ}, \psi=-40^{\circ}$ for glycine in the selected monomers shown in Fig. 1C). Importantly, the mutation leads to the translocation of the $\beta 6-\beta 7$ loop and, as a consequence, to a relative shift of the $\beta 7$ toward the $\beta 6$ strand. These rearrangements lead to the occlusion of the cGMP binding cavity that is formed by residues in $\beta 6, \beta 7$, and $\alpha \mathrm{C}$ (Zhou and Siegelbaum, 2007) and a reduction of the cGMP binding site volume. Both effects may impair cGMP binding and, hence, channel activation. We tested this hypothesis by introducing the mutation into the CNBD of CNGA1, a subunit that unlike CNGB1a is able to form functional homomeric channels. The mutation had no consequences on protein expression levels (Fig. 1D). However, as predicted, CNGA1GV channels were functionally inactive in the presence of a saturating cGMP concentration (1 mM) (Fig. 1E). Even at $10 \mathrm{~mm}$ cGMP CNGA1GV channels did not give rise to any detectable currents (not shown). Moreover, coexpression of CNGB1a or the olfactory CNGB1b subunit that lacks the GARP domain failed to rescue channel activity (Fig. $1 E$ ).

We next asked which effects the GV mutation in CNGB1a exerts on the heteromeric channel complex. To this end, we coexpressed CNGA1 together with either wildtype or mutant CNGB1a and compared the properties of the resulting currents with currents induced by CNGA1 alone. Surprisingly, currents obtained after coexpression of CNGA1 and CNGB1aGV did not exhibit typical CNGB1a-mediated features, but were indistinguishable from currents of homomeric CNGA1 channels (Fig. 2A-D). While single-channel currents of heteromeric CNG channels are characterized by very brief open-closed transitions (singlechannel flicker), single-channel currents obtained after coexpression of CNGA1 and CNGBlaGV displayed long openings, which is typical of homomeric CNGA1 channels (Fig. $2 A$ ). In addition, the strong outwardly rectifying current-voltage relation of channels obtained after coexpression of CNGA1 and CNGB1aGV in the presence of extracellular divalent ions was virtually identical to that of the homomeric CNGA1 channel, but clearly differed from that of weakly rectifying wild-type heteromeric channels (Fig. 2B). In contrast to wild-type CNGB1a, 
CNGB1aGV did not increase $\mathrm{Ca}^{2+} / \mathrm{CaM}$ sensitivity if coexpressed with CNGA1 (Fig. 2C). Probably the most indicative feature resulting from the presence of the CNGB1 subunit is the generation of a high-affinity site for block by L-cisdiltiazem (Chen et al., 1993). Indeed, unlike homomeric CNGA1 channels, heteromeric CNGA1/CNGB1a channels were efficiently blocked by this agent. By contrast, currents obtained after coexpression with CNGB1aGV were not sensitive to this drug (Fig. 2D). The total absence of CNGBlamediated properties in conjunction with the complete correspondence with the biophysical properties of homomeric CNGA1 currents indicated that currents measured after cotransfection of CNGA1 and CNGB1aGV were purely caused by CNGA1 homomers. A possible explanation for this result would be that after cotransfection of CNGA1 and CNGB1aGV, only homomeric CNGA1 channels are present in the membrane because CNGA1/CNGB1aGV heteromers are not assembled, are rapidly degraded, or fail to be transported to the plasma membrane. However, cell surface biotinylation experiments using membrane impermeable amine-reactive biotin showed that both wild-type and mutant channels were present at the membrane of transfected cells (Fig. 2E). In addition, CNGB1aGV could be coimmunoprecipitated with CNGA1, indicating the formation of stable heteromers (Fig. 2F). Finally, assembly of CNGA1 with CNGB1aGV was verified by FRET (Fig. 2G).

\section{The GARP domain of CNGB1a is} responsible for functional silencing of mutant CNG channel heteromers

Another explanation for the selective detection of homomeric CNGA1 currents in HEK293 cells transfected with CNGA1 and CNGBlaGV may be that homomeric and mutant heteromeric CNG channels do coexist in the plasma membrane, but in contrast to homomeric CNGA1 channels, mutant heteromers cannot be activated by cGMP and, thus, do not contribute any measurable current. In an attempt to identify structural determinants conferring an inhibitory action of CNGB1aGV, we introduced the GV mutation into the olfactory CNGB1b subunit that differs from CNGB1a by lacking the GARP domain and coexpressed this new mutant with CNGA1 (Fig. 3A). Surprisingly, L-cisdiltiazem-sensitive currents were now obtained with both wildtype and mutant CNGB1b subunits. Sensitivity to L-cis-diltiazem was also consistently observed when the GV mutation was introduced into the native olfactory CNG channel complex consisting of CNGA2/CNGA4 and CNGB1b (Fig. $3 A$ ). In both combina-

A

C

$\mathrm{F}$

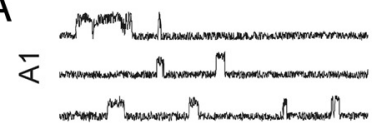

$\mathrm{B}$
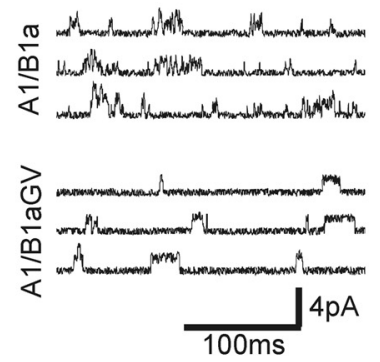

D

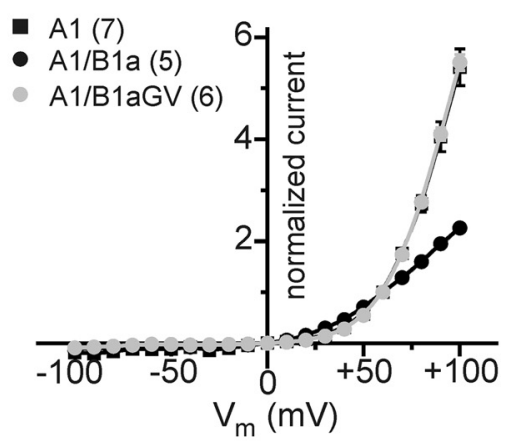

E
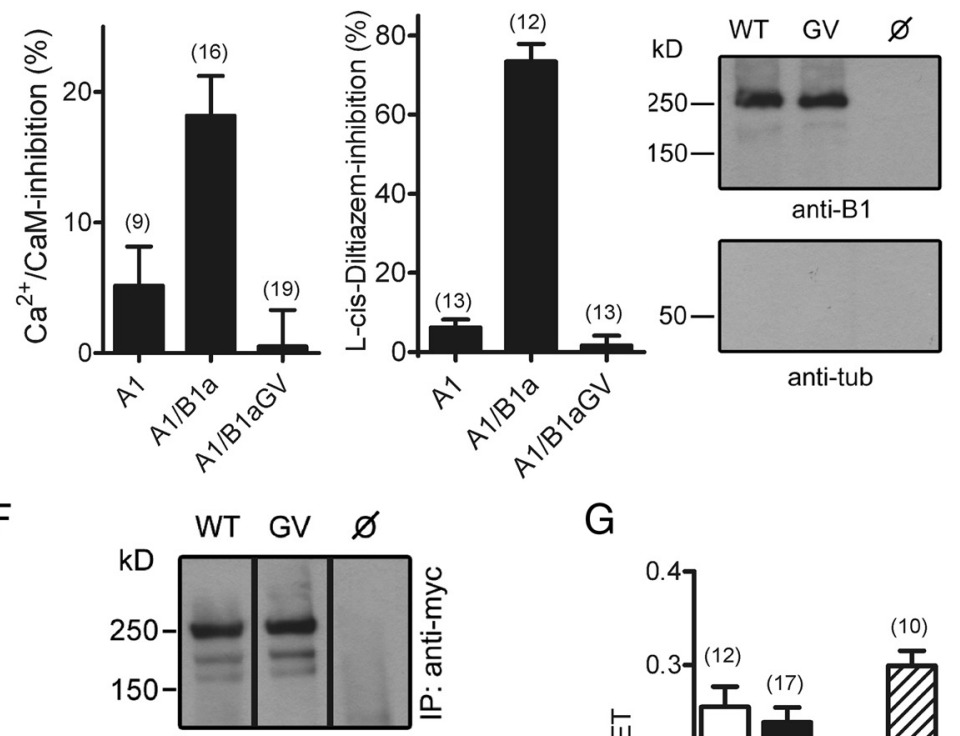

IB: anti-B1

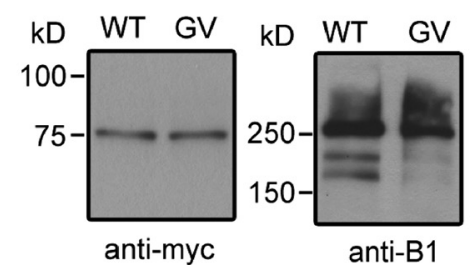

G

Figure 2. Heteromeric CNGA1/CNGB1aGV channels are present in the plasma membrane but are functionally inactive. $A$, Single-channel currents induced at $+60 \mathrm{mV}$ by $3 \mu \mathrm{m} \mathrm{CGMP}$ in excised patches of HEK293 cells transfected with CNGA1 (A1), CNGA1/CNGB1a (A1/B1a), or CNGA1/CNGB1aGV (A1/B1aGV). B, Current-voltage relation of A1, A1/B1a, and A1/B1aGV in the presence of $2 \mathrm{~mm} \mathrm{Ca}^{2+}$ and $1 \mathrm{~mm} \mathrm{Mg}^{2+}$ in the extracellular solution. Current was normalized to the current at $+60 \mathrm{mV}$. $C$, Inhibition of $\mathrm{A} 1, \mathrm{~A} 1 / \mathrm{B} 1 \mathrm{a}$, and $\mathrm{A} 1 / \mathrm{B} 1 \mathrm{aGV}$ currents by $250 \mathrm{~nm}$ calmodulin $/ 100 \mu \mathrm{M} \mathrm{Ca}{ }^{2+}$ in the bath solution. Currents were induced at $+80 \mathrm{mV}$ by $100 \mu \mathrm{m}$ CGMP. D, L-cis-Diltiazem $(10 \mu \mathrm{m})$ block of A1, A1/B1a, and A1/B1aGV currents induced by $300 \mu \mathrm{m} \mathrm{cGMP}$ at $+80 \mathrm{mV}$. $\boldsymbol{E}$, Cell-surface biotinylation of nontransfected ( $($ ) and CNGA1/CNGB1a- (WT) and CNGA1/CNGB1aGV- (GV) transfected HEK293 cells. Cells were biotinylated for $30 \mathrm{~min}$ at $4^{\circ} \mathrm{C}$ and probed with anti-B1 (top) or anti-tubulin (tub, bottom) antibodies. $\boldsymbol{F}$, Coimmunoprecipitation from lysates of HEK293 cells cotransfected with myc-tagged CNGA1 and wild-type CNGB1a or CNGB1aGV, respectively. Top, Immunoprecipitation (IP) using anti-myc for pulldown and anti-B1 for detection. Bottom, Western blots showing the starting material for the co-IP probed with anti-myc (left) or anti-B1 antibodies (right). G, N-FRET ratios calculated from HEK293 cells cotransfected with CNGA1-CFP and YFP-CNGB1a (WT) or YFP-CNGB1aGV, respectively. HEK293 cells transfected with CFP and YFP-CNGB1a or a CFP-YFP tandem were used for the calculation of negative (neg) and positive (pos) control N-FRET values, respectively. The number of experiments is given in parentheses. All values are given as mean $\pm \mathrm{SE}$.

tions the slightly reduced L-cis-sensitivity of B1bGV-containing channels was not significantly different from the respective wildtype channels $(p>0.05)$ (Fig. 3A). Like for CNGA1, the wildtype but not the mutant CNGB1a subunit induced L-cis-diltiazem sensitivity when coexpressed together with CNGA2 (Fig. 3A). 

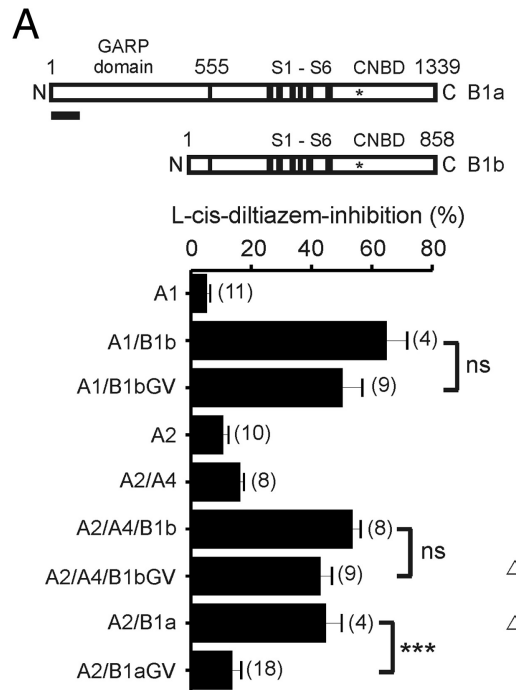

B
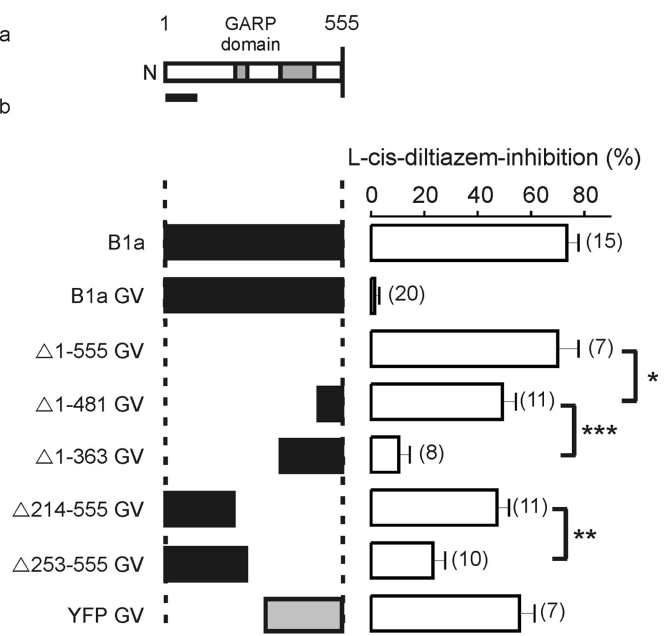

Figure 3. The GARP domain of CNGB1a is responsible for functional silencing of mutant CNG channel heteromers. $A$, The GV mutation does not impair channel function in the context of the olfactory CNGB1b isoform lacking the GARP domain. Top, Schematic representation of CNGB1a and CNGB1b subunits. The position of the GV mutation is highlighted by an asterisk. Bottom, Summary graph showing the L-cis-diltiazem sensitivity of various homomeric or heteromeric channels. Currents were elicited by $300 \mu \mathrm{m} \mathrm{CGMP}$ at $+80 \mathrm{mV}$. L-cis-diltiazem was used at $50 \mu \mathrm{m}$ for A2, A2/A4, A2/A4/B1b, and $\mathrm{A} 2 / \mathrm{A} 4 / \mathrm{B} 1 \mathrm{bGV}$ and at $10 \mu \mathrm{m}$ for all other channels. $B$, Mutagenesis screening of sequences conferring the inhibitory effect of the GARP domain (amino acids 1-555). Top, Diagram showing the $N$ terminus of CNGB1a (amino acids 1-555). The diagram is in scale with the bars in the bottom panel illustrating the $\mathrm{N}$-terminal truncation constructs. The two sequences conferring most of the inhibitory effect are highlighted in gray. Bottom, Graphic overview on N-terminal truncation constructs and summary graph comparing the effect (\% inhibition) of $10 \mu \mathrm{M} \mathrm{L-cis-diltiazem} \mathrm{on} \mathrm{cGMP} \mathrm{currents} \mathrm{(300} \mu \mathrm{m}$, $+80 \mathrm{mV}$ ) measured from HEK293 cells cotransfected with CNGA1 and various N-terminal truncated CNGB1aGV mutants or a B1aGV chimera in which the $\mathrm{N}$ terminus was replaced by the yellow fluorescent protein (YFP). The number of experiments is given in parentheses. ${ }^{*} p<0.05,{ }^{* *} p<0.01,{ }^{* *} p<0.001, \mathrm{~ns}=$ not statistically significant. All values are given as mean \pm SE. Scale bar, 100 aa. S1-S6, Transmembrane helices.

A

GARP2 $\quad$\begin{tabular}{rr}
1 & 314 \\
\hline
\end{tabular}

B1a\#1-555 N שـ
B1a\#556-1339 GV

B

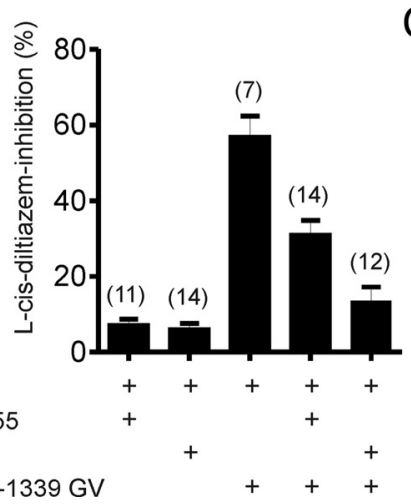

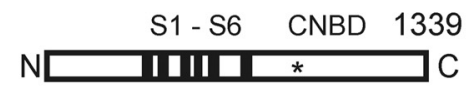

C

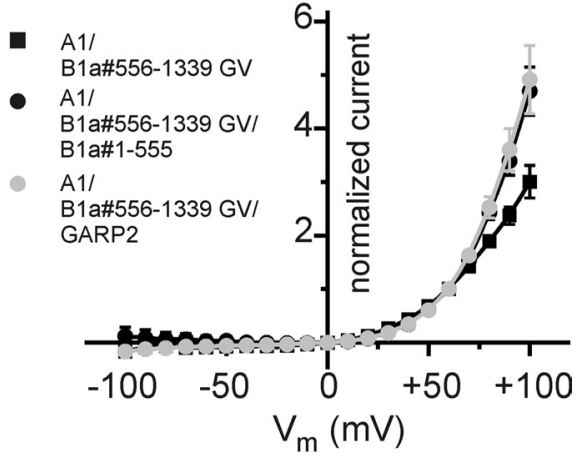

A1

B1a\#1-555

GARP2

B1a\#556-1339 GV
Figure 4. GARP2 and the GARP domain of CNGB1a act as autonomous channel inhibitors. $A$, Schematic representation of GARP2, the N terminus of CNGB1a containing the complete GARP domain (B1a\#1-555), and the truncated B1a\#556-1339 GV channel. B, GARP2 and B1a\#1-555 reduce the L-cis-diltiazem inhibition of A1/B1a\#556-1339 GV channels. Shown is percentage inhibition by $10 \mu \mathrm{M} \mathrm{L-cis-diltiazem} \mathrm{of} \mathrm{currents} \mathrm{obtained} \mathrm{after} \mathrm{cotransfection} \mathrm{of} \mathrm{CNGA1} \mathrm{with} \mathrm{various} \mathrm{constructs} \mathrm{as} \mathrm{indicated.} \mathrm{C,}$ GARP2 and B1a\#1-555 increase the outward rectification of A1/B1a\#556-1339 GV currents in the presence of extracellular divalents. Shown is the current-voltage relation of A1/B1a\#556-1339 GV, A1/B1a\#556-1339 GV/GARP2, and A1/B1a\#556-1339 GV/B1a\#1-555 in the presence of $2 \mathrm{~mm} \mathrm{Ca}^{2+}$ and $1 \mathrm{~mm} \mathrm{Mg}^{2+}$ in the extracellular solution. Current was normalized to the current at $+60 \mathrm{mV}$. The number of experiments is given in parentheses, and all values are given as mean $\pm \mathrm{SE}$.
These results indicated that the GARP domain determinates the dominant-negative effect of CNGB1aGV. Analysis of mutants with deletions in the GARP domain identified two regions (residues 214-253 and $364-481$ of rat CNGB1a) that confer most of the inhibitory effect (Fig. $3 B$ ). Both regions do not overlap with the repeat regions (R1-R4) within the GARP domain (Sugimoto et al., 1991; Körschen et al., 1995; Colville and Molday, 1996). The second domain is probably highly charged since it harbors a large number of glutamate (82) and lysine (25) residues. Fusion of an unrelated peptide (YFP) to the $\mathrm{N}$ terminus of CNGB1bGV did not result in significant loss in L-cis-diltiazem sensitivity (Fig. $3 B)(p>0.05)$.

\section{The GARP domain acts inhibitory if coexpressed as separate protein}

To test whether GARPs can act autonomously, we coexpressed either the fulllength GARP domain (B1a\#1-555) or GARP2, which contains the N-terminal half of the GARP domain, together with CNGA1 and the GV mutant CNGBla truncation construct (B1a\#556-1339GV) (see diagram in Fig. 4A). As already shown, CNGA1/B1a\#556-1339GV currents were sensitive to L-cis-diltiazem (Fig. $4 B)$. By contrast, coexpression of either full-length GARP or GARP2 abolished the block by L-cis-diltiazem (Fig. $4 B$ ) and led to an increase of the outward rectification in the presence of extracellular divalents (Fig. 4C). The inhibitory effect of GARP did not depend on $\mathrm{Ca}^{2+}$, since addition of $100 \mu \mathrm{M} \mathrm{Ca}^{2+}$ to the bath solution did not significantly change the ability of GARP2 to reduce the L-cis-diltiazem sensitivity of CNGA1/CNGB1a\#556-1339GV channels [block by L-cis-diltiazem: $17.9 \pm 3.3 \%(n=$ $10)$ at $100 \mu \mathrm{M} \mathrm{Ca}^{2+}$ and $13.2 \pm 4.03 \%(n=$ 12) at $\left.0 \mu \mathrm{M} \mathrm{Ca}^{2+}, p>0.05\right]$.

In conclusion, these findings strongly suggested that the GARP domain exerts its inhibitory effect even if it is not covalently linked to the CNGB1 core. In agreement with this hypothesis, the GARP domain (B1a\#1-555) formed stable protein complexes with both CNGA1 (Fig. 5B) and either mutant or wild-type CNGB1a\# 556-1339 (Fig. 5C). In line with the electrophysiological experiments, we found that GARP2 also coimmunoprecipitated with CNGB1a\#556-1339 (Fig. 5D). Interestingly, we also found that peripherin-2, another protein known to interact with GARP2 and CNGB1a (Poetsch et al., 2001; Batra-Safferling et al., 2006), did also interact with CNGB1a\#556-1339 (Fig. 5D). Thus, peripherin-2 seems to in- 
A
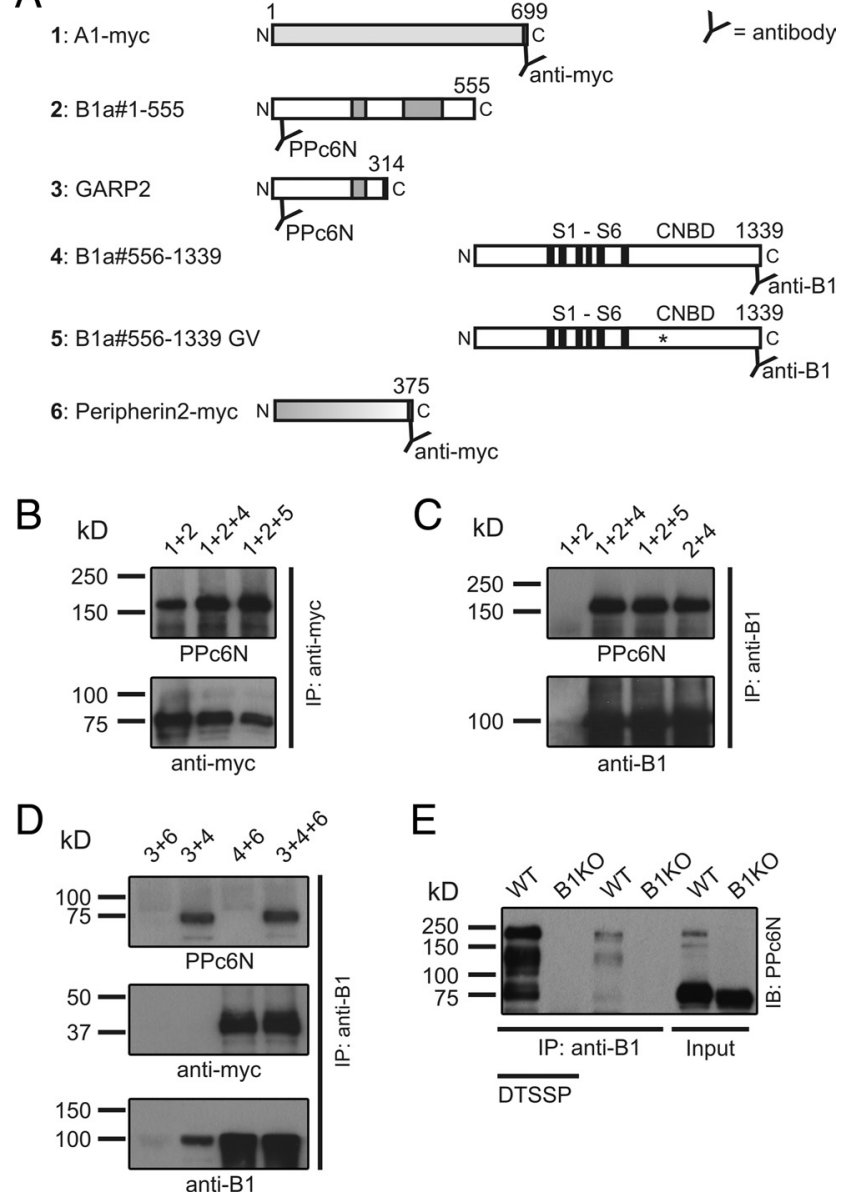

E

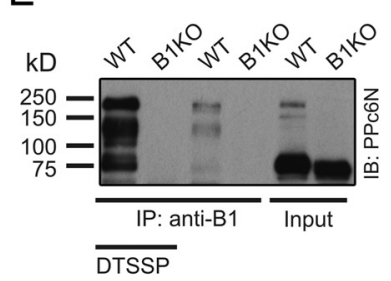

Figure 5. Soluble GARPs and the GARP domain of CNGB1a interact with the channel domain. $A$, Schematic representation of constructs used for the experiments shown in $\boldsymbol{B}-\boldsymbol{D}$. Each construct is given a number (1-6) that is used for the lane headings in $\boldsymbol{B}-\boldsymbol{D}$. $\boldsymbol{B}-\boldsymbol{D}$, Coimmunoprecipitations from HEK293 cells expressing various combinations of the constructs shown in $\boldsymbol{A} . \boldsymbol{B}, \boldsymbol{C}$, The N terminus of CNGB1a (B1a\#1-555, $\sim 160 \mathrm{kDa}$ ) coimmunoprecipitates with myc-tagged CNGA1 $(\sim 75 \mathrm{kDa})(\boldsymbol{B})$ and truncated B1a\#556-1339 WT or GV ( $\sim 100 \mathrm{kDa}$, respectively) (C). D, CNGB1a\#556-1339 coimmunoprecipitates with GARP2 ( $\sim 70 \mathrm{kDa})$ (top) and myc-tagged peripherin-2 ( $\sim 40 \mathrm{kDa}$ ) (middle). $\boldsymbol{E}$, CNGB1a coimmunoprecipitates with GARP1 ( $\sim 130 \mathrm{kDa}$ ) and GARP2 from wild-type mouse retinal lysates. The interaction can be detected with (first lane) or without (third lane) the aid of the reversible chemical crosslinker DTSSP and is absent in $\mathrm{CNGB1}^{-1-}$ mice (B1KO; lanes 2 and 4). Anti-B1, Rabbit polyclonal antibody directed against the C terminus of CNGB1a. PPc6N, Rabbit polyclonal antibody directed against the N terminus of CNGB1a. IB, Immunoblot; IP, immunoprecipitation.

teract with multiple domains of CNGB1a (e.g., the GARP and the channel domain). However, peripherin-2 was not able to compete for GARP2 binding to CNGB1a\#556-1339 (Fig. 5D), suggesting the presence of different binding sites for these two proteins. Finally, both native GARP isoforms (GARP1 and GARP2) did coimmunoprecipitate with CNGB1a from mouse retinal tissue lysates (Fig. 5E).

\section{A functional CNBD in CNGB1a is required to remove tonic inhibition imposed by the GARP domain}

Our data indicated that the GARP domain binds to both CNGA1 and CNGB1 subunits in the heteromeric CNG channel complex. In wild-type heteromers, the identified GARP-channel interaction does not interfere with cGMP-dependent activation. By contrast, in the presence of the GV mutation, the GARP domain completely shuts down channel activity. Based on our MD sim-
A
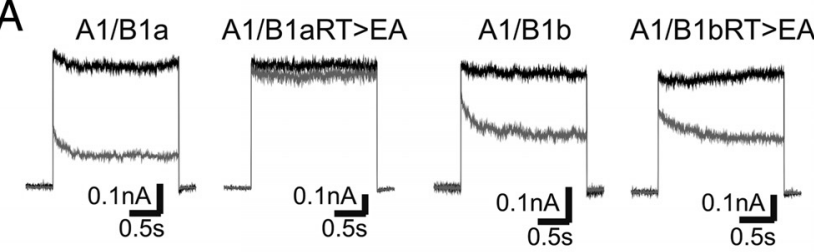

B

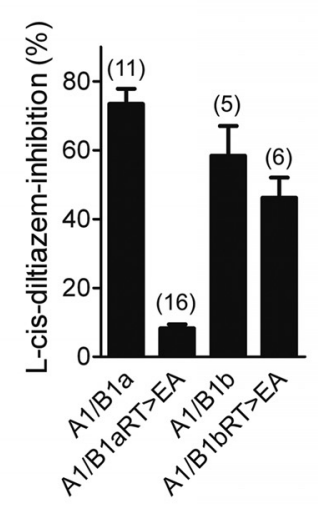

C
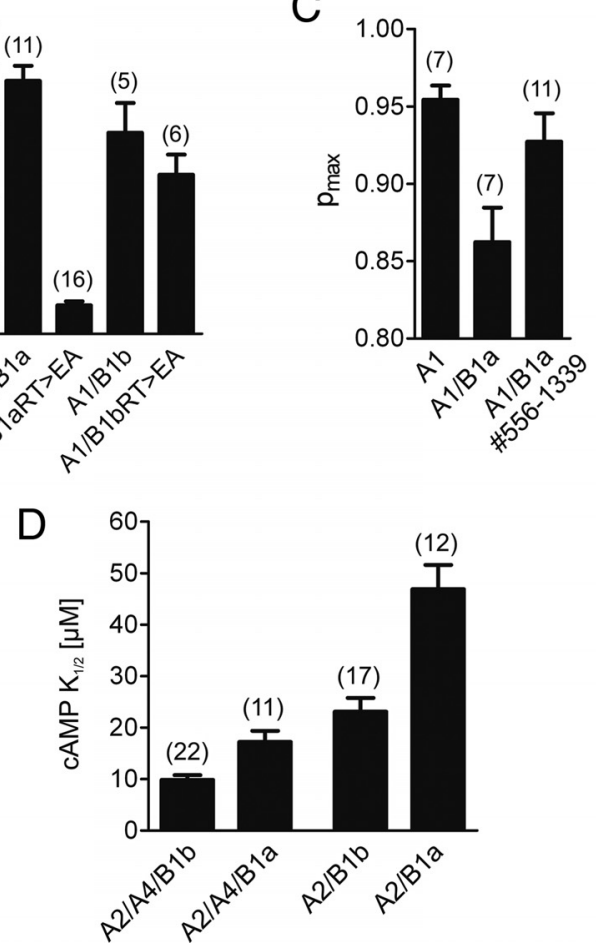

Figure 6. The GARP domain silences heteromeric CNG channels containing CGMP-binding deficient CNGB1a subunits. $\boldsymbol{A}$, Representative currents traces recorded at $+80 \mathrm{mV}$ after application of $300 \mu \mathrm{m}$ cGMP in the absence (black traces) or presence of $10 \mu \mathrm{m}$ L-cis-diltiazem (gray traces) are shown. $\boldsymbol{B}$, Summary graph comparing the L-cis-diltiazem sensitivity of heteromeric channels containing wild-type and cyclic nucleotide-binding deficient (RT>EA) B1a or B1b subunits. RT $>$ EA, Substitution of arginine and threonine within the $\beta 7$-sheet of the CNBD by glutamate and alanine, respectively. C, Summary graph comparing the maximal open probability for $\mathrm{GMP}\left(P_{\text {max }}\right)$ of wild-type homomeric A1, heteromeric A1/B1a, and A1/B1a\#556-1339 channels. $D$, Summary graph comparing the CAMP sensitivity of heteromeric channels containing B1a or B1b subunits. The number of experiments is given in parentheses. All values are given as mean $\pm S E$.

ulations (Fig. 1C), we speculated that the inhibitory effect of GARP might be causally linked to the inability of CNGB1aGV to interact with cGMP. To test this hypothesis, we analyzed the effects of GARP in the presence of a double mutation in the $\beta 7$ strand of the CNBD (R1042E and T1043A, hereafter referred to as $\mathrm{RT}>\mathrm{EA}$ ) that is well known to abolish cyclic nucleotide binding in CNG and HCN channels (Zagotta et al., 2003; Zhou and Siegelbaum, 2007). As anticipated, in the backbone of CNGB1b the double mutation did not interfere with the formation of functional heteromeric (L-cis-diltiazem-sensitive) channels (Fig. $6 A, B)$. However, when introduced into CNGB1a, the mutation fully blocked heteromeric channel activity, and only the residual homomeric (L-cis-diltiazem insensitive) CNGA1 channel was detected (Fig. 6A,B). Thus, with respect to its functional effect on heteromeric channels containing or lacking the GARP domain, the $\mathrm{RT}>\mathrm{EA}$ mutation was indistinguishable from the GV mutation. 
A
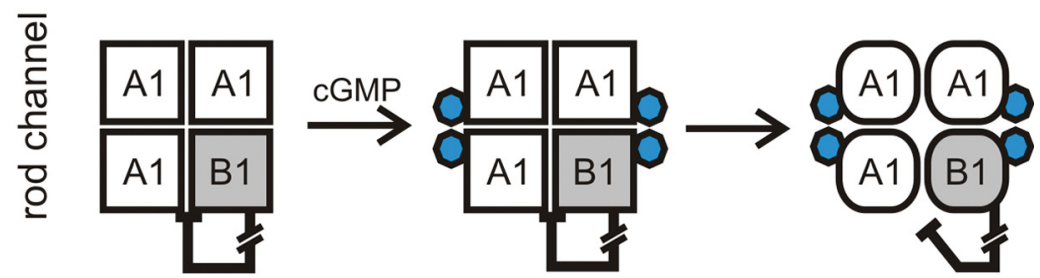

B
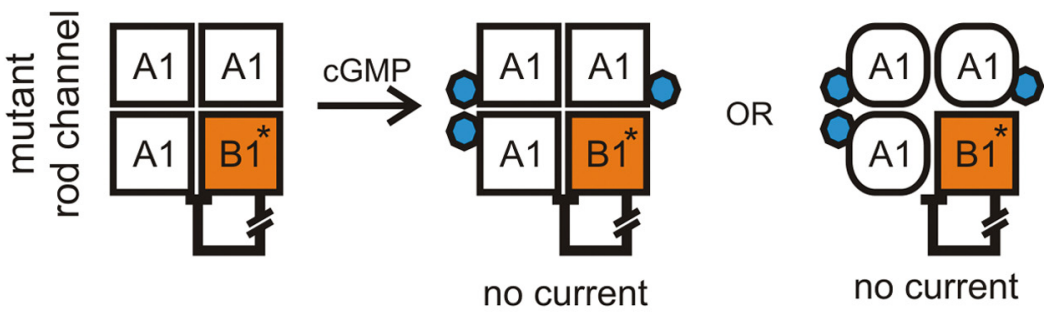

C
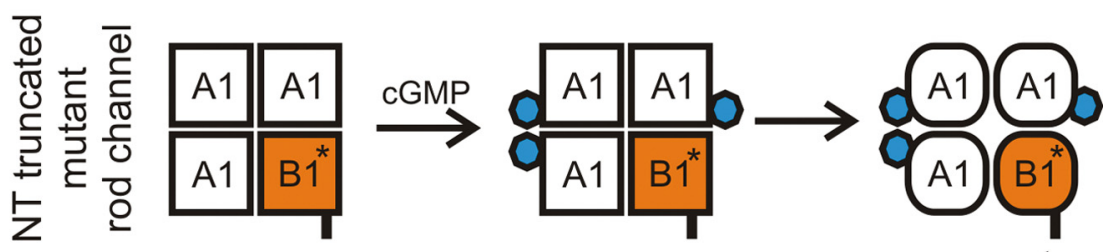

current

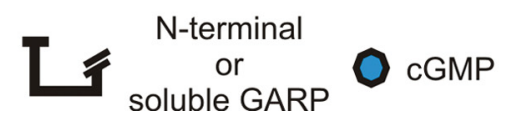

Figure 7. Model of the action of the GARP domain in wild-type $(A)$ and mutant heteromeric $C N G$ channels $(B, C)$. $A$, In wild-type rod channels, GARP (N-terminal or soluble) serves as a gating inhibitor. Binding of CGMP to the CNGB1a subunit releases the tonic inhibition mediated by the GARP domain giving rise to channel opening. For reasons of clarity, the contemplable intermediates (i.e., if only one or two CGMP are bound to CNGA1) are not included in this model. $\boldsymbol{B}$, The GV mutation in CNGB1a prevents CGMP binding to this subunit. Therefore, the tonic inhibition of GARP is not removed and the channels remain in the closed state. $C$, If GARP is not present the GV mutant heteromeric channels are able to open in the presence of GGMP.

The GARP domain lowers the open probability of rod CNG channels and decreases the cAMP sensitivity of olfactory CNG channels

The experiments shown so far indicated that the GARP domain blocks gating of heteromeric $\mathrm{CNG}$ channels containing a CNGB1 subunit deficient for cGMP binding. We were wondering whether the GARP domain could also interfere with the gating of wild-type heteromeric channels containing a functional CNBD. To address this important issue, we compared the open probability for cGMP $\left(P_{\max }\right)$ of CNGA1/CNGBla with that of CNGA1/ CNGB1a\#556-1339 channels lacking GARP (Fig. 6C). Indeed, channels containing the GARP domain had a significantly lower $P_{\max }$ than channels lacking this domain $\left[P_{\max (\mathrm{cGMP})}\right.$ : CNGA1/ CNGB1a $=0.86 \pm 0.02(n=7)$ (similar to data from Pagès et al., 2000), CNGA1/CNGB1a\#556-1339=0.93 $\pm 0.02(n=11), p<$ 0.05]. This finding strongly supports the notion that the GARP domain acts as gating inhibitor in the rod photoreceptor channel. To test whether the inhibitory action of the GARP domain is transferable to another system, we analyzed the effect of GARP on the sensitivity of the olfactory CNG channel to its physiological ligand CAMP. We coexpressed the olfactory CNGA2 and CNGA4 subunits with the CNGB1a subunit from rod photoreceptors and compared the CAMP sensitivity with the native olfactory channel containing the CNGB1b subunit. In support of a general inhibitory action of the GARP domain on CNG channel activation, the CNGA2/CNGA4/CNGB1a heterotetramers showed a decreased
cAMP sensitivity as compared to the native olfactory CNG channel $\left[K_{1 / 2 \text { (cAMP): }}\right.$ : $\mathrm{CNGA} 2 / \mathrm{CNGA} 4 / \mathrm{CNGB} 1 \mathrm{a}=17.20 \pm 2.19$ $(n=11), \mathrm{CNGA} 2 / \mathrm{CNGA} 4 / \mathrm{CNGB} 1 \mathrm{~b}=$ $9.85 \pm 0.91(n=22), p<0.001$ ] (Fig. $6 D)$. To rule out a contribution of CNGA4, we repeated the experiment without this subunit and obtained a similar effect after comparison of heteromeric CNGA2/ CNGB1a and CNGA2/CNGB1b channels $\left[K_{1 / 2 \text { (cAMP) }}:\right.$ CNGA2/CNGB1a $=46.90 \pm$ $4.70(n=12)$, CNGA2/CNGB1b $=$ $23.10 \pm 2.65(n=17), p<0.0001$ ] (Fig. $6 D)$.

\section{Discussion}

In this study, we have examined the role of the CNGB1a subunit in the cGMPdependent activation process of the rod photoreceptor CNG channel. The CNBD of CNGB1a contains all canonical residues required for high-affinity cGMP binding (Chen et al., 1993; Körschen et al., 1995; Biel et al., 1996; Kaupp and Seifert, 2002; Biel and Michalakis, 2009) and was shown in photoaffinity labeling experiments to bind cGMP (Brown et al., 1995). While these findings suggest that CNGB1a contributes to channel activation, the analysis of the role of CNGB1a in this process has been hampered by the fact that CNGB1a does not form functional homomeric channels. We have shed new light on this issue by analyzing a naturally occurring mutation in CNGB1a of RP patients (GV mutation) that impairs the structural integrity and/or cGMP binding capability of the CNBD. We found that this mutation exerts a strong dominantnegative effect leading to a complete functional silencing of heteromeric channels. Surprisingly, however, the impact of the GV mutation was strictly dependent on the presence of the proximal part of the $\mathrm{N}$ terminus of CNGB1a (GARP domain) and was not present in the short CNGB1b subunit expressed in olfactory CNG channels. Unlike homomeric CNGA1 or heteromeric CNGA1/ CNGB1aGV channels, channels containing CNGB1bGV displayed high sensitivity to block by L-cis-diltiazem, which is the pharmacological hallmark of a functional CNGB1 subunit. The different effect of the two CNGB1 isoforms was also observed for another extensively characterized mutation impairing cGMP binding (RT $>$ EA mutation) and was found when CNGB1a/b were assembled with another CNG A subunit (CNGA2). Two important conclusions can be drawn from this finding. First, in the absence of the GARP domain binding of cGMP to CNGB1 is not required for principal activation of heteromeric channels. This is in good agreement with recent studies showing that in the olfactory channels, only the CNGA2 subunit of the tetrameric complex must be functional to activate the channel (Waldeck et al., 2009) and that binding of only two cyclic nucleotides is sufficient for maximal opening of these channels (Biskup et al., 2007). Second, however, the presence of the GARP domain strongly interferes with the activation process, transforming cyclic nucleotide binding to CNGB1 from a nonessential process to a prerequisite of channel activation. An inhibitory effect of the GARP 
domain on channel gating is supported by our findings that wildtype rod channels have a lower open probability than rod channels lacking the GARP domain and that this domain impairs the cAMP sensitivity of olfactory CNG channels. We therefore propose that GARP is an inhibitory domain that controls channel activation (Fig. $7 A-C$ ). In the native rod $\mathrm{CNG}$ channel, the GARP domain interacts with the channel complex (CNGA1 and/or CNGB1) in its unliganded state. Binding of cGMP to CNGA1 and CNGB1a relieves the inhibitory effect of GARP, leading to channel opening (Fig. 7A). In channels containing a CNGBla subunit that is deficient for cGMP binding, the inhibitory impact of GARP cannot be removed, and CNGBla stays locked in its closed state. This prevents the opening transition of the tetrameric channel complex even if cGMP is bound to the three CNGA1 subunits (Fig. $7 B$ ). In the absence of the GARP domain, there is no longer a constraint on channel gating. Now, binding of cGMP to the CNGA1 subunits is sufficient for full activation (Fig. 7C). The exact molecular mechanism underlying the action of the GARP domain remains to be determined. However, our in vitro and in vivo coimmunoprecipitation experiments indicate that GARP can directly bind to CNGB1, suggesting that a direct proteinprotein interaction underlies the effect of GARP. The GARP domain was shown to have a flexible, largely unordered structure in solution (Batra-Safferling et al., 2006). We propose that like other unstructured proteins such as bacterial FlgM (Dedmon et al., 2002), GARP can gain structure in the presence of specific binding partners. The identification of two sequences within the GARP domain that are responsible for most of its inhibitory effect supports the notion that GARP specifically interacts with the CNG channel. We also show that GARP acts inhibitory if expressed as separate protein, indicating that it forms an autonomous folding unit. The latter finding is important since it indicates that also soluble GARP1 and GARP2 that are coexpressed together with CNGB1a in rod outer segments can inhibit channel gating. It was proposed that the GARP domain of CNGBla tethers the heteromeric CNG channel to the disc rim of outer segments by interacting with peripherin-2 (Poetsch et al., 2001; Batra-Safferling et al., 2006). Our finding that GARP2 could still bind to the channel when peripherin-2 was also present indicates that distinct domains within GARP are involved in binding to peripherin-2 and the channel, respectively. This also suggests that the major GARP isoform in rod photoreceptors, GARP2, that contains one of the sequences conferring channel inhibition and is present in rods at $\sim 25$-fold molar excess over CNGB1a (Batra-Safferling et al., 2006) may contribute most of the inhibitory effect in vivo. This hypothesis is corroborated by our finding that GARP2 has a similar negative effect on channel gating as B1a\#1-555. However, we also found that more GARP1 than GARP2 coimmunoprecipitates with the channel from mouse retinal lysates (see lane 3 in Fig. 5E). Based on the higher endogenous levels of GARP2 (Körschen et al., 1999), one would have expected more GARP2 than GARP1 to be detected in the coimmunoprecipitation experiments. The reason for this discrepancy is not clear, but it may be explained by differences in affinity and/or additional binding partners for the two GARP isoforms within the native $\mathrm{CNG}$ channel complex.

Currently, one can only speculate on physiological implications arising from the GARP-mediated inhibition of the unliganded CNG channel. Given that homomeric CNGA1 channels have a very small but detectable spontaneous open probability (Ruiz and Karpen, 1997; Tibbs et al., 1997), the inhibitory effect of GARP may have evolved to reduce current noise resulting from openings of CNG channels in the absence of cGMP to the lowest possible levels. Interestingly, GARP2 was also reported to bind to and suppress the basal activity of the rod phosphodiesterase (PDE6) (Pentia et al., 2006). This would result in higher basal cGMP levels and thus increased activity of the CNG channel. Together with our results, this suggests that in the absence of light stimulus GARP2 may act to prevent spontaneous activation of two key elements of the phototransduction cascade, the CNG channel and the phosphodiesterase to facilitate single-photon sensitivity.

Finally, our study explains the clinical phenotype of patients carrying the GV mutation. Since heteromeric CNGA1/ CNGB1aGV channels are inactive, rod photoreceptors of the patients cannot respond to light-induced changes in the cGMP concentration. Importantly and unlike in HEK293 cells, in rod photoreceptors CNG channel activity cannot be conferred by homomeric CNGA1 channels since these channels may not be targeted to outer segments in sufficient amounts (Hüttl et al., 2005; Zhang et al., 2009). Thus, the phenotype of CNGB1aGV patients is comparable to a total knock-out of the CNGB1 subunit that also results in RP (Hüttl et al., 2005; Zhang et al., 2009).

\section{References}

Bareil C, Hamel CP, Delague V, Arnaud B, Demaille J, Claustres M (2001) Segregation of a mutation in CNGB1 encoding the beta-subunit of the rod cGMP-gated channel in a family with autosomal recessive retinitis pigmentosa. Hum Genet 108:328-334.

Batra-Safferling R, Abarca-Heidemann K, Körschen HG, Tziatzios C, Stoldt M, Budyak I, Willbold D, Schwalbe H, Klein-Seetharaman J, Kaupp UB (2006) Glutamic acid-rich proteins of rod photoreceptors are natively unfolded. J Biol Chem 281:1449-1460.

Berendsen HJC, Postma JPM, van Gunsteren WF, Hermans PAKJ, Dixon R, Cornell W, Fox T, Chipot C, Pohorille A (1981) Interaction models for water in relation to protein hydration. In: Intermolecular forces (Wilkinson A, Weiner P, van Gunsteren WF, eds), p 331. Dordrecht, Germany: Reidel.

Biel M, Michalakis S (2009) Cyclic nucleotide-gated channels. Handb Exp Pharmacol 191:111-136.

Biel M, Altenhofen W, Hullin R, Ludwig J, Freichel M, Flockerzi V, Dascal N, Kaupp UB, Hofmann F (1993) Primary structure and functional expression of a cyclic nucleotide-gated channel from rabbit aorta. FEBS Lett 329:134-138

Biel M, Zong X, Ludwig A, Sautter A, Hofmann F (1996) Molecular cloning and expression of a modulatory subunit of the cyclic nucleotide-gated cation channel. J Biol Chem 271:6349-6355.

Biskup C, Kusch J, Schulz E, Nache V, Schwede F, Lehmann F, Hagen V, Benndorf K (2007) Relating ligand binding to activation gating in CNGA2 channels. Nature 446:440-443.

Brown RL, Gramling R, Bert RJ, Karpen JW (1995) Cyclic GMP contact points within the $63-\mathrm{kDa}$ subunit and a $240-\mathrm{kDa}$ associated protein of retinal rod cGMP-activated channels. Biochemistry 34:8365-8370.

Chen TY, Peng YW, Dhallan RS, Ahamed B, Reed RR, Yau KW (1993) A new subunit of the cyclic nucleotide-gated cation channel in retinal rods. Nature 362:764-767.

Colville CA, Molday RS (1996) Primary structure and expression of the human beta-subunit and related proteins of the rod photoreceptor cGMPgated channel. J Biol Chem 271:32968-32974.

Darden T, York D, Pedersen L (1993) Particle mesh Ewald: an $\mathrm{N} \cdot \log (\mathrm{N})$ method for Ewald sums in large systems. J Chem Phys 98:10089-10092.

Dedmon MM, Patel CN, Young GB, Pielak GJ (2002) FlgM gains structure in living cells. Proc Natl Acad Sci U S A 99:12681-12684.

Eswar N, Webb B, Marti-Renom MA, Madhusudhan MS, Eramian D, Shen MY, Pieper U, Sali A (2007) Comparative protein structure modeling using MODELLER. Curr Protoc Protein Sci 2:2.9.

Flynn GE, Black KD, Islas LD, Sankaran B, Zagotta WN (2007) Structure and rearrangements in the carboxy-terminal region of SpIH channels. Structure 15:671-682.

Gordon SE, Zagotta WN (1995) A histidine residue associated with the gate of the cyclic nucleotide-activated channels in rod photoreceptors. Neuron 14:177-183. 
Griessmeier K, Cuny H, Rötzer K, Griesbeck O, Harz H, Biel M, Wahl-Schott C (2009) Calmodulin is a functional regulator of Cav1.4 L-type Ca2+ channels. J Biol Chem 284:29809-29816.

Haber-Pohlmeier S, Abarca-Heidemann K, Körschen HG, Dhiman HK, Heberle J, Schwalbe H, Klein-Seetharaman J, Kaupp UB, Pohlmeier A (2007) Binding of Ca2 + to glutamic acid-rich polypeptides from the rod outer segment. Biophys J 92:3207-3214.

Hüttl S, Michalakis S, Seeliger M, Luo DG, Acar N, Geiger H, Hudl K, Mader R, Haverkamp S, Moser M, Pfeifer A, Gerstner A, Yau KW, Biel M (2005) Impaired channel targeting and retinal degeneration in mice lacking the cyclic nucleotide-gated channel subunit CNGB1. J Neurosci 25:130-138.

Ildefonse M, Crouzy S, Bennett N (1992) Gating of retinal rod cation channel by different nucleotides - comparative-study of unitary currents. J Membr Biol 130:91-104.

Kaupp UB, Seifert R (2002) Cyclic nucleotide-gated ion channels. Physiol Rev 82:769-824.

Kaupp UB, Niidome T, Tanabe T, Terada S, Bönigk W, Stühmer W, Cook NJ, Kangawa K, Matsuo H, Hirose T, Miyata T, Numa S (1989) Primary structure and functional expression from complementary DNA of the rod photoreceptor cyclic GMP-gated channel. Nature 342:762-766.

Körschen HG, Illing M, Seifert R, Sesti F, Williams A, Gotzes S, Colville C, Müller F, Dosé A, Godde M, Molday L, Kaupp UB, Molday RS (1995) A $240 \mathrm{kDa}$ protein represents the complete beta subunit of the cyclic nucleotide-gated channel from rod photoreceptor. Neuron 15:627-636.

Körschen HG, Beyermann M, Müller F, Heck M, Vantler M, Koch KW, Kellner R, Wolfrum U, Bode C, Hofmann KP, Kaupp UB (1999) Interaction of glutamic-acid-rich proteins with the cGMP signalling pathway in rod photoreceptors. Nature 400:761-766.

Luna EJ (2001) Biotinylation of proteins in solution and on cell surfaces. Curr Protoc Protein Sci 3:3.6.

Much B, Wahl-Schott C, Zong X, Schneider A, Baumann L, Moosmang S, Ludwig A, Biel M (2003) Role of subunit heteromerization and $\mathrm{N}$-linked glycosylation in the formation of functional hyperpolarizationactivated cyclic nucleotide-gated channels. J Biol Chem 278:4378143786.

Pagès F, Ildefonse M, Ragno M, Crouzy S, Bennett N (2000) Coexpression of alpha and beta subunits of the rod cyclic GMP-gated channel restores native sensitivity to cyclic AMP: role of D604/N1201. Biophys J 78:1227-1239.

Pentia DC, Hosier S, Cote RH (2006) The glutamic acid-rich protein-2 (GARP2) is a high affinity rod photoreceptor phosphodiesterase (PDE6)binding protein that modulates its catalytic properties. J Biol Chem 281:5500-5505.

Poetsch A, Molday LL, Molday RS (2001) The cGMP-gated channel and related glutamic acid-rich proteins interact with peripherin-2 at the rim region of rod photoreceptor disc membranes. J Biol Chem 276:4800948016.
Ruiz ML, Karpen JW (1997) Single cyclic nucleotide-gated channels locked in different ligand-bound states. Nature 389:389-392.

Sautter A, Biel M, Hofmann F (1997) Molecular cloning of cyclic nucleotide-gated cation channel subunits from rat pineal gland. Brain Res Mol Brain Res 48:171-175.

Sautter A, Zong X, Hofmann F, Biel M (1998) An isoform of the rod photoreceptor cyclic nucleotide-gated channel beta subunit expressed in olfactory neurons. Proc Natl Acad Sci U S A 95:4696-4701.

Shabb JB, Corbin JD (1992) Cyclic nucleotide-binding domains in proteins having diverse functions. J Biol Chem 267:5723-5726.

Sugimoto Y, Yatsunami K, Tsujimoto M, Khorana HG, Ichikawa A (1991) The amino acid sequence of a glutamic acid-rich protein from bovine retina as deduced from the cDNA sequence. Proc Natl Acad Sci U S A 88:3116-3119.

Sunderman ER, Zagotta WN (1999) Sequence of events underlying the allosteric transition of rod cyclic nucleotide-gated channels. J Gen Physiol 113:621-640.

Tibbs GR, Goulding EH, Siegelbaum SA (1997) Allosteric activation and tuning of ligand efficacy in cyclic-nucleotide-gated channels. Nature 386:612-615.

Van Der Spoel D, Lindahl E, Hess B, Groenhof G, Mark AE, Berendsen HJ (2005) GROMACS: fast, flexible, and free. J Comput Chem 26:17011718.

Waldeck C, Vocke K, Ungerer N, Frings S, Möhrlen F (2009) Activation and desensitization of the olfactory cAMP-gated transduction channel: identification of functional modules. J Gen Physiol 134:397-408.

Weitz D, Ficek N, Kremmer E, Bauer PJ, Kaupp UB (2002) Subunit stoichiometry of the CNG channel of rod photoreceptors. Neuron 36:881-889.

Xia Z, Liu Y (2001) Reliable and global measurement of fluorescence resonance energy transfer using fluorescence microscopes. Biophys J 81:2395-2402.

Zagotta WN, Olivier NB, Black KD, Young EC, Olson R, Gouaux E (2003) Structural basis for modulation and agonist specificity of $\mathrm{HCN}$ pacemaker channels. Nature 425:200-205.

Zhang Y, Molday LL, Molday RS, Sarfare SS, Woodruff ML, Fain GL, Kraft TW, Pittler SJ (2009) Knockout of GARPs and the beta-subunit of the rod cGMP-gated channel disrupts disk morphogenesis and rod outer segment structural integrity. J Cell Sci 122:1192-1200.

Zheng J, Trudeau MC, Zagotta WN (2002) Rod cyclic nucleotide-gated channels have a stoichiometry of three CNGA1 subunits and one CNGB1 subunit. Neuron 36:891-896.

Zhong H, Molday LL, Molday RS, Yau KW (2002) The heteromeric cyclic nucleotide-gated channel adopts a 3A:1B stoichiometry. Nature 420:193-198.

Zhou L, Siegelbaum SA (2007) Gating of HCN channels by cyclic nucleotides: residue contacts that underlie ligand binding, selectivity, and efficacy. Structure 15:655-670. 